International Journal of Engineering \& Technology, 7 (4.10) (2018) 316-318
International Journal of Engineering \& Technology
SPC
Website: $w$ ww.sciencepubco.com/index.php/IJET
Research paper

\title{
Diabetes Disease Analysis Using Rough Soft Set
}

\author{
A.Manimaran ${ }^{1}$, B. Praba ${ }^{2}$, G. Deepa ${ }^{3, *}$, V. M. Chandrasekaran ${ }^{4}$, Krishnamoorthy Venkatesan $^{5}$ \\ ${ }^{1,3,4}$ School of Advanced Sciences, VIT, Vellore, Tamilnadu, India \\ ${ }^{2}$ SSN College of Engineering, Kalavakkam, Chennai, Tamilnadu, India \\ ${ }^{5}$ College of Natural Sciences, Arba Minch University, Ethiopia \\ *Corresponding authorE-mail: ${ }^{1}$ marans2011@gmail.com, ${ }^{2}$ prabab@ssn.edu.in, ${ }^{3, *}$ deepa.g@vit.ac.in, ${ }^{4}$ vmcsn@yahoo.com, \\ ${ }^{5}$ krishnamoorthy.venkatesan@amu.edu.et
}

\begin{abstract}
Diabetes is a noteworthy medical issue in both modern and creating nations, and its frequency is rising apparently. It is a metabolic disease in which the person who has been affected will have high blood glucose or high blood sugar. It is mainly because of inadequate production of insulin or the body's cells do not respond to insulin. In some special cases it may be due to both the reasons. This disease causes a lot of health issues in humans' life. Rough set and soft set theory plays a major role for dealing with uncertainty and it has been applied in many fields. In this paper we aim at finding the age group of people in which maximum diabetes mellitus occurs using the concept of rough soft set and rough soft decision set.
\end{abstract}

Keywords: Lower approximation, Upper approximation, Fuzzy set, Rough set, Soft set, Rough soft set

\section{Introduction}

Nowadays, in our daily life due to lack of physical work and change in food habits causes many problems in our human health. In particular our immune system is affected due to change in our food habits. Diabetes mellitus (DM) is a chronic disease that is characterized by high blood glucose. It happens when a body can't deliver or react legitimately to insulin which is expected to control glucose and it expands the dangers of creating kidney sickness, visual deficiency, nerve harm, vein harm also it adds to coronary illness. Many authors contributed their research towards the medical diagnosis problem. Deepa et al. [1] discussed about the medical diagnosis problem using IF set in terms of shortest distance measure in 2018. Feng et al. [2] described the concept of the combination of soft set with fuzzy set and rough sets in 2010. The authors Ghosh and Samanta [3] described some basic properties of rough sets and rough soft groups in 2013. Manimaran et al. [4, 5, 6, 13] discussed a rough set approach for medical diagnosis, an algorithm for deduction of time to detect alzheimer's disease and the concept of rough semiring on the set of all rough sets in 2015 also a review of medical diagnosis is given by the same authors in fuzzy environment in 2016. In 2018, the same authors [7] described an analysis for skin disease using IF set. Meng et al. [8] introduced the concept of soft rough fuzzy set and soft fuzzy rough sets in 2011. For dealing uncertainty, Pawlak and Moldstov $[9,12]$ introduced a mathematical tool called rough set theory in 1982 and soft set theory in 1999 respectively. Temurats et al. [14] described a comparative study on diabetes disease diagnostic by using multi-layer neural network in 2009. Wu et al. [15] introduced a model using data mining for predicting type 2 diabetes mellitus in 2018. Zadeh [16] introduced the concept of fuzzy sets for dealing vagueness in 1965. Zhang [17] introduced a rough set approach to intuitionistic fuzzy soft set in decision making techniques in 2012. In this direction, we present an analysis for identi- fying the diabetic disease among the different age group using rough soft decision set.

\section{Preliminaries}

Diabetes mellitus is a chronic disease which has risen as a worldwide epidemic. Diabetes is a metabolic condition of having higher than normal blood sugar levels. There are different reasons why people get high blood glucose levels and so there are several types of diabetes exist. They are,

1. Type 1 diabetes (due to b-cell destruction, usually leading to absolute insulin deficiency)

2 . Type 2 diabetes (due to a progressive insulin secretory defect on the background of insulin resistance)

3. Gestational diabetes mellitus (GDM) (diabetes diagnosed in the second or third trimester of pregnancy that is not clearly overt diabetes)

4. Specific types of diabetes due to other causes, e.g., monogenic diabetes syndromes (such as neonatal diabetes and maturity-onset diabetes of the young), diseases of the exocrine pancreas (such as cystic fibrosis), and drug- or chemical-induced diabetes (such as in the treatment of HIV/AIDS or after organ transplantation).

Definition 2.1 (Fuzzy Set) [16]

Let $X$ be a non-empty set. A fuzzy set $A$ from $X$ is defined as $A=\left\{\left(x, \mu_{A}(x)\right): x \in X\right\}$ where $\mu_{A}(x): X \rightarrow[0,1]$ is the membership function of the fuzzy set $A$.

\section{Example 2.1 [16]}

Consider the temperature of a patient in degree Celsius. Let $X=\{36.5,37,37.5,38,38.5,39,39.5\}$. The fuzzy set $A$ "High temperature" may be defined as, 
$A=\{(0,36.5),(0,37),(0.1,37.5),(0.5,38),(0.8,38.5),(1,39),(1,39.5)\}$ tion operators on soft sets. If $\underline{R}(\Omega)=\bar{R}(\Omega)$ then the soft set $\Omega$ is

where the numbers $0,0.1,0.5,0.8$ and 1 express the degree to which the corresponding temperature is high.

\section{Definition 2.2 (Rough Set) $[4,12,13]$}

Let $U$ be the finite universal set and $R$ be an equivalence relation on $U$. The pair $(U, R)$ is called a Pawlak approximation space and $R$ will generate a partition $U / R=\left\{[x]_{R}: x \in U\right\}$ where $[x]_{R}$ is the equivalence class with respect to $R$ containing $x$ then the pair $R S(X)=(\underline{R}(X), \bar{R}(X))$ is called as rough set of an arbitrary subset $X$ of $U$ where $\underline{R}(X)=\left\{x \in U:[x]_{R} \subseteq X\right\}$ is called the lower approximation and $\bar{R}(X)=\left\{x \in U:[x]_{R} \cap X \neq \phi\right\}$ is called the upper approximation of $X$ with respect to $(U, R)$.

Note that, if $\underline{R}(X)=\bar{R}(X)$ then $X$ is called definable in $(U, R)$ otherwise $X$ is called rough set.

\section{Example 2.2 [4, 12, 13]}

Let $U=\left\{p_{1}, p_{2}, p_{3}, p_{4}, p_{5}, p_{6}\right\}$ be the patients suffering from flu with the following symptoms as attributes $\left\{\mathrm{e}_{1}, \mathrm{e}_{2}, \mathrm{e}_{3}, \mathrm{e}_{4}\right\}$ where $\mathrm{e}_{1}, \mathrm{e}_{2}, \mathrm{e}_{3} \& \mathrm{e}_{4}$ stands for headache, body pain, vomit and cold respectively. Let $\left[p_{1}\right]_{R}=\left\{p_{1}, p_{3}, p_{4}\right\} \quad, \quad\left[p_{2}\right]_{R}=\left\{p_{2}\right\} \quad$, $\left[p_{5}\right]_{R}=\left\{p_{5}, p_{6}\right\}$ be the equivalence classes of the relation $R=\left\{e_{1}, e_{2}, e_{3}, e_{4}\right\} \quad$ on $U$. For an arbitrary subset $X=\left\{p_{1}, p_{2}, p_{4}, p_{6}\right\}$ of $U$, then the lower approximation of $X$ is $\underline{R}(X)=\left\{p_{2}\right\}$ and the upper approximation of $X$ is $\bar{R}(X)=\left\{p_{1}, p_{2}, p_{3}, p_{4}, p_{5}, p_{6}\right\}$ then the rough set corresponding to $X$ on $(U, R)$ is $R S(X)=\left(\left\{p_{2}\right\},\left\{p_{1}, p_{2}, p_{3}, p_{4}, p_{5}, p_{6}\right\}\right)$.

\section{Definition 2.3 (Soft Set) $[8,9]$}

Let $F_{A}: E \rightarrow P(U)$ be the approximate function where $P(U)$ be the power set of the initial inverse $U$ and $A \subseteq E$ where $E$ be the set of parameters then the ordered pair $\left(F_{A}, E\right)=\left\{\left(e, F_{A}(e)\right): e \in E \& F_{A}(e) \in P(U)\right\}$ and $F_{A}(e)=0$ for $e \notin A$ is called as a soft set. Also the set $F_{A}(e)$ is called eapproximate set for all $e \in E$.

\section{Example 2.3 [8, 9]}

Let $U$ be the universal set contains seven cars $\left\{c_{1}, c_{2}, c_{3}, c_{4}, c_{5}, c_{6}, c_{7}\right\}$ and $E=\left\{e_{1}, e_{2}, e_{3}, e_{4}\right\}$ be the set of parameters where each $e_{i}$ stands for spacious, costly, beautiful and good design in the market for $i=1,2,3$ and 4 respectively. Now the soft set $(F, E)$ is described by the nature of the car. Suppose that $F\left(e_{1}\right)=\left\{c_{2}, c_{3}, c_{5}\right\}, F\left(e_{2}\right)=\left\{c_{1}, c_{4}\right\}, F\left(e_{3}\right)=\left\{c_{1}, c_{3}, c_{6}\right\}$ and $F\left(e_{4}\right)=\left\{c_{2}, c_{3}, c_{6}, c_{7}\right\}$. Then the soft set is a parameterized family $\left\{F\left(e_{i}\right): 1 \leq i \leq 4\right\}$ of subsets of $U$ and gives us a collection of approximate descriptions of an object. $F\left(e_{4}\right)=\left\{c_{2}, c_{3}, c_{6}, c_{7}\right\}$ stands for the cars $c_{2}, c_{3}, c_{6} \& c_{7}$ are good in design in the market.

\section{Definition 2.4 (Rough Soft Set) [3]}

Let $(U, R)$ be the Pawlak approximation space and $\Omega=(F, A)$ be a soft set over $U$. The lower and upper approximations of $\Omega$ in $(U$, $R$ ) are denoted by $\underline{R}(\Omega)=\left(F_{*}, A\right)$ and $\bar{R}(\Omega)=\left(F^{*}, A\right)$, which are soft sets over $U$ with the set valued mapping given by,

$F_{*}(x)=\underline{R}(F(x))=\left\{y \in U:[y]_{R} \subseteq F(x)\right\}$ and

$F^{*}(x)=\bar{R}(F(x))=\left\{y \in U:[y]_{R} \cap F(x) \neq \phi\right\}$ for all $x \in A$. The operators $\underline{R} \& \bar{R}$ are called the lower and upper rough approxima- said to be definable otherwise $\Omega$ is called rough soft set.

\section{Proposed Model}

To find the age group at which maximum possibility of diabetes disease occur using rough soft decision set.

\section{Definition 3.1 (Rough Soft Decision Set)}

Let $R(\Omega)=\left(F_{*}(x), F^{*}(x)\right)$ be the rough soft set then the rough soft decision set is denoted by $R^{D}(\Omega)$ and it is defined as $R^{D}(\Omega)=\left\{R_{D}(x) / x: x \in U\right\}$ where $R_{D}(x)=\frac{\left|F_{*}\left(P_{i}\right)\right|+\left|F^{*}\left(P_{i}\right)\right|}{2|E|}$ where each $P_{i} \in X \subseteq U$.

\subsection{Analysis Using RSD}

\section{Step 1:}

Let $I=(U, A)$ be an information system where $U=\left\{x_{1}, x_{2}, x_{3}, x_{4}, x_{5}, x_{6}\right\}$ be the finite universal set of patients where $x_{1}: 0-5$ years $, x_{2}: 6-15$ years $, x_{3}: 16-24$ years, $x_{4}: 25-45$ years $, x_{5}: 45-65$ years \& $x_{6}:$ Above 65 and $A=\left\{y_{1}, y_{2}, y_{3}, y_{4}, y_{5}\right\}$ be the set of attributes where each $y_{i}$ stands for excess thirst, bed wetting, increased fatigue, blurred vision and weight fluctuation respectively for $1 \leq i \leq 5$. Each attribute $y_{i}: U \rightarrow V_{\alpha}$ for $y_{i} \in A$ where $V_{\alpha}$ is the set of fuzzy membership values. The information system is given below,

Table - 1

\begin{tabular}{|l|l|l|l|l|l|}
\hline $\mathrm{A} / \mathrm{U}$ & $y_{1}$ & $y_{2}$ & $y_{3}$ & $y_{4}$ & $y_{5}$ \\
\hline$x_{1}$ & 0.8 & 0.1 & 0.2 & 0.3 & 0.6 \\
\hline$x_{2}$ & 0.8 & 0.7 & 0.1 & 0.25 & 0.2 \\
\hline$x_{3}$ & 0.7 & 0.8 & 0.3 & 0.1 & 0.3 \\
\hline$x_{4}$ & 1.0 & 0.9 & 0.6 & 0.3 & 0.4 \\
\hline$x_{5}$ & 0.7 & 0.8 & 0.3 & 0.1 & 0.3 \\
\hline$x_{6}$ & 0.8 & 0.7 & 0.1 & 0.25 & 0.2 \\
\hline
\end{tabular}

and the equivalence classes are given below,

$\left[x_{1}\right]_{R}=\left\{x_{1}\right\},\left[x_{2}\right]_{R}=\left\{x_{2}, x_{6}\right\}$

$\left[x_{3}\right]_{R}=\left\{x_{3}, x_{5}\right\}$ and $\left[x_{4}\right]_{R}=\left\{x_{4}\right\}$

Step 2:

Average of $x_{i}{ }^{\prime} s$ :

Table - 2

\begin{tabular}{|l|l|l|l|l|l|}
\hline$\overline{x_{1}}$ & $\overline{x_{2}}$ & $\overline{x_{3}}$ & $\overline{x_{4}}$ & $\overline{x_{5}}$ & $\overline{x_{6}}$ \\
\hline 0.4 & 0.41 & 0.44 & 0.64 & 0.44 & 0.41 \\
\hline
\end{tabular}

Step 3:

Now, define $P$ such that

$P=\left\{\bar{x}_{i}{ }^{\prime} s: \overline{x_{i}} \geq \overline{x_{j}}\right.$ for $\left.i \neq j, j=1,2,3,4,5\right\}$

Table - 3

\begin{tabular}{|l|l|l|}
\hline \multicolumn{3}{|c|}{ Table - 3 } \\
\hline$\overline{x_{i}}$ & \multicolumn{1}{|c|}{$P^{\prime} s$} & Corresponding $x_{i}{ }^{\prime} s$ \\
\hline$x_{1}$ & $\phi$ & $\phi$ \\
\hline$x_{2}$ & $\overline{x_{1}}, \overline{x_{6}}$ & $x_{1}, x_{6}$ \\
\hline
\end{tabular}




\begin{tabular}{|l|l|l|}
\hline$x_{3}$ & $\overline{x_{1}}, \overline{x_{2}}, \overline{x_{5}}, \overline{x_{6}}$ & $x_{1}, x_{2}, x_{5}, x_{6}$ \\
\hline$x_{4}$ & $\overline{x_{1}}, \overline{x_{2}}, \overline{x_{3}}, \overline{x_{5}}, \overline{x_{6}}$ & $x_{1}, x_{2}, x_{3}, x_{5}, x_{6}$ \\
\hline$x_{5}$ & $\overline{x_{1}}, \overline{x_{2}}, \overline{x_{3}}, \overline{x_{6}}$ & $x_{1}, x_{2}, x_{3}, x_{6}$ \\
\hline$x_{6}$ & $\overline{x_{1}}, \overline{x_{2}}$ & $x_{1}, x_{2}$ \\
\hline
\end{tabular}

Step 4:

Let $U=\left\{x_{1}, x_{2}, x_{3}, x_{4}, x_{5}, x_{6}\right\}$ be the universal set and the equivalence classes are $\left[x_{1}\right]_{R}=\left\{x_{1}\right\},\left[x_{2}\right]_{R}=\left\{x_{2}, x_{6}\right\}, \quad\left[x_{3}\right]_{R}=\left\{x_{3}, x_{5}\right\}$ and $\left[x_{4}\right]_{R}=\left\{x_{4}\right\}$ where $R$ is the equivalence relation on $U$. Let $\Omega=(F, A)$ be a soft set over $U$ and let $A=U$.

Let $F: A \rightarrow P(U)$ is a set valued function defined by $F(x)=\left\{x_{i} \in U: x_{i} \rho x_{j} \Leftrightarrow \overline{x_{i}} \geq \overline{x_{j}} \forall x_{j} \in A\right\}$.Then the elements of the soft set are $F\left(x_{1}\right)=\phi, F\left(x_{2}\right)=\left\{x_{1}, x_{6}\right\}, F\left(x_{3}\right)=\left\{x_{1}, x_{2}, x_{5}, x_{6}\right\}$, $F\left(x_{4}\right)=\left\{x_{1}, x_{2}, x_{3}, x_{5}, x_{6}\right\}$ and $F\left(x_{6}\right)=\left\{x_{1}, x_{2}\right\}$ and the lower and upper approximations of this soft sets are given below.

Table -4

\begin{tabular}{|c|c|}
\hline$F_{*}\left(x_{i}\right)$ & $F^{*}\left(x_{i}\right)$ \\
\hline$\phi$ & $\phi$ \\
\hline$\left[x_{1}\right]_{R}$ & {$\left[x_{1}\right]_{R} \cup\left[x_{2}\right]_{R}$} \\
\hline$\left[x_{1}\right]_{R} \cup\left[x_{2}\right]_{R}$ & {$\left[x_{1}\right]_{R} \cup\left[x_{2}\right]_{R} \cup\left[x_{3}\right]_{R}$} \\
\hline$\left[x_{1}\right]_{R} \cup\left[x_{2}\right]_{R} \cup\left[x_{3}\right]_{R}$ & {$\left[x_{1}\right]_{R} \cup\left[x_{2}\right]_{R} \cup\left[x_{3}\right]_{R}$} \\
\hline$\left[x_{1}\right]_{R} \cup\left[x_{2}\right]_{R}$ & {$\left[x_{1}\right]_{R} \cup\left[x_{2}\right]_{R} \cup\left[x_{3}\right]_{R}$} \\
\hline$\left[x_{1}\right]_{R}$ & {$\left[x_{1}\right]_{R} \cup\left[x_{2}\right]_{R}$} \\
\hline
\end{tabular}

\section{Step 5:}

Rough soft decision set:

The values of $R_{D}(x)$ is given below,

Table -5

\begin{tabular}{|l|l|l|l|}
\hline \multicolumn{1}{|c|}{$x_{i}{ }^{\prime} s$} & $\left|F_{*}\left(x_{i}\right)\right|$ & $\left|F^{*}\left(x_{i}\right)\right|$ & $\frac{\left|F_{*}\left(P_{i}\right)\right|+\left|F^{*}\left(P_{i}\right)\right|}{2|E|}$ \\
\hline$x_{1}$ & 0 & 0 & 0 \\
\hline$x_{2}$ & 1 & 3 & 0.4 \\
\hline$x_{3}$ & 3 & 5 & 0.8 \\
\hline$x_{4}$ & 5 & 5 & 1 \\
\hline$x_{5}$ & 3 & 5 & 0.8 \\
\hline$x_{6}$ & 1 & 3 & 0.4 \\
\hline
\end{tabular}

The rough soft decision set has been attained by using the definition

$$
R^{D}(\Omega)=\left\{0\left|x_{1}, 0.4\right| x_{2}, 0.8\left|x_{3}, 1.0\right| x_{4}, 0.8\left|x_{5}, 0.4\right| x_{6}\right\}
$$

\section{Step 6:}

Now it is clear that $R_{D}(x)=1.0$ is maximum. Therefore,

we conclude that age group of 25 to 45 years most likely to suffer in diabetic problem using rough soft decision set.

\section{Conclusion}

Giving analytic guide to diabetes infection by utilizing an arrangement of information that contains just medical information acquired without any advanced medical equipment will help the quantity of individuals who need to find the sickness. In this paper, we introduced a diabetes infection demonstrative strategy by using rough soft decision set since there are many methods available to diagnose diabetic disease in humans. But in our method we have discussed that which age group of people affected maximum with diabetic disease using rough soft decision set. Hence we conclude that diabetes is affected maximum among the age-group of 25-45 using rough soft decision set.

\section{References}

[1] G. Deepa, B. Praba, A. Manimaran, V. M. Chandrasekaran and Raja Kumar. K (2018), Medical Diagnosis using Intuitionistic Fuzzy set in Terms Shortest Distance Measure, Research Journal of Pharmacy and Technology, 11(3), 949-952.

[2] F. Feng, C. Li, B. Davvaz \& M.I. Ali (2010), Soft sets combined with fuzzy sets and rough sets: a tentative approach, Soft Computing, 14(9), $899-911$

[3] J. Ghosh \& T. K. Samanta (2013), Rough soft sets and rough soft groups, Journal of Hyperstructures, 2(1), 18 - 29.

[4] A. Manimaran, V. M. Chandrasekaran and Aishwarya Asesh (2015), Rough Set Approach for an Efficient Medical Diagnosis System, International Journal Of Pharmacy \& Technology, 7(1), 8049-8060

[5] A. Manimaran, V. M. Chandrasekaran, Keshav Gupta, Megha Kanwar and P. M. Karthick(2015), A new algorithm for deduction of time to detect Alzheimer's disease, Journal of Chemical and Pharmaceutical Research, 7(12), 197 - 205.

[6] A.Manimaran, V.M.Chandrasekaran, B.Praba, A Review of Fuzzy Environmental Study in Medical Diagnosis System, Research journal of Pharmacy and Technology, 9 (2) (2016), 177 - 184

[7] A. Manimaran, B. Praba, V. M. Chandrasekaran, Karan Agrawal and Akanksha Miharia (2018), Skin disease analysis using Intuitionistic Fuzzy Set, Research Journal of Pharmacy and Technology, 11(1), 79-82.

[8] D. Meng, X. Zhang \& K. Qin (2011), Soft rough fuzzy sets and soft fuzzy rough sets, Computers \& mathematics with applications, 62(12), 4635 - 4645

[9] D. Molodtsov (1999), Soft set theory - First results, Comput. Math. Appl., 37, $19-31$.

[10] M. Muthumeenakshi and P. Muralikrishna, A Study on SFPM Analysis Using Fuzzy Soft Set, International Journal of Pure and Applied Mathematics, 94 (2) (2014), 207 - 213.

[11] Nilashi, M., Ibrahim, O., Dalvi, M., Ahmadi, H., \& Shahmoradi, L. (2017). Accuracy Improvement for Diabetes Disease Classification: A Case on a Public Medical Dataset, Fuzzy Information and Engineering, 9(3), 345 - 357.

[12] Z. Pawlak (1982), Rough sets, Int. J. Comput. Inform. Sci. 11, 341 356.

[13] B. Praba, V. M. Chandrasekaran and A. Manimaran(2015), Semiring on Rough Sets, Indian Journal of Science and Technology, 8(3), $280-286$

[14] H. Temurtas, N. Yumusak \& F. Temurtas (2009). A comparative study on diabetes disease diagnosis using neural networks. Expert Systems with applications, 36(4), 8610-8615.

[15] Wu, H., Yang, S., Huang, Z., He, J., \& Wang, X. (2018). Type 2 diabetes mellitus prediction model based on data mining. Informatics in Medicine Unlocked, 10, 100 - 107.

[16] L. A. Zadeh, Fuzzy Sets, Information and Control, 8(1965), 338 353.

[17] Zhang, Z. (2012). A rough set approach to intuitionistic fuzzy soft set based decision making, Applied Mathematical Modelling, 36(10), 4605 - 4633. 